\title{
The critical behavior of the BCS order parameter: a straightforward derivation
}

\author{
Roland Köberle ${ }^{* 1}$ \\ ${ }^{1}$ Universidade de São Paulo, Departamento de Física, São Carlos, SP, Brasil.
}

Received on April 6, 2021. Revised on May 3, 2021. Accepted on May 4, 2021.

Textbooks on Solid State Physics, such as [1 3], include a mandatory chapter on Superconductivity. Usually the basic item to start with is the famous Bardeen-CooperSchrieffer model(BCS) [4. One of the main results concerns the way in which the superconducting order parameter $\boldsymbol{\Delta}(T)$ vanishes at the critical temperature $T_{c}$, namely as

$$
\boldsymbol{\Delta}(T) \sim B\left(T_{c}-T\right)^{1 / 2}
$$

where the prefactor $B$ is a non-universal coefficient and the exponent has the classical value $\alpha=1 / 2^{1}$

Then one may read that this is a standard result for any mean-field theory, although the student may wonder, why it does not follow straightforwardly from the model?

Yet in the literature this outstandingly simple statement is obtained in a rather roundabout manner. Furthermore $\alpha$ and $B$ are computed only in the weakcoupling limit $\hbar \omega_{D} \gg k_{B} T_{c}$, where $\omega_{D}$ is the Debye frequency. This is certainly an aesthetically not very pleasing situation and I doubt the student really wants to grind through the approximations just to get this simple result.

The following lines show a little trick straightening out this situation. It will hopefully find its way to the textbooks.

In the BCS theory the order-parameter $\boldsymbol{\Delta}(T)$ satisfies the non-linear integral equation ${ }^{2}$

$$
1=g \int_{0}^{\hbar \omega_{D}} d \epsilon \frac{\tanh \left(\frac{\beta E}{2}\right)}{2 E}
$$

with $E=\sqrt{\epsilon^{2}+\Delta^{2}}, \beta=1 / k_{B} T$ and $g$ is some coupling constant.

\footnotetext{
* Correspondence email address: rk@ifsc.usp.br

${ }^{1}$ Although traditionally the exponent is referred to as $\beta=1 / 2$, we use $\alpha$ to avoid confusions with $\beta=1 / k_{B} T$

${ }^{2}$ See e.g [3] equation(23.20) or [2] equation (6.28)
}

We extract the critical behavior of the order parameter straightforwardly and without approximations. For this purpose we choose $\boldsymbol{\Delta}$ to be real and parametrize it as

$$
\Delta(\beta)=a\left(\frac{\beta-\beta_{c}}{\beta_{c}}\right)^{\alpha} ; \beta \sim \beta_{c}
$$

This yields for the derivative $\partial_{\beta} \Delta^{2} \equiv \frac{\partial \Delta^{2}}{\partial \beta}$ :

$$
\lim _{T \rightarrow T_{c}} \partial_{\beta} \Delta^{2}= \begin{cases}0 & \alpha>1 / 2 \\ a^{2} / \beta_{c} & \alpha=1 / 2 \\ \infty & \alpha<1 / 2\end{cases}
$$

The non-linear integral equation (2) for the order parameter has the solution $\boldsymbol{\Delta}\left(\beta, \omega_{D}, g\right)$, depending on three parameters. Substituting this solution into equation (2) yields an identity. Differentiating this identity with respect to $\beta$ easily yields the following relation

$$
\partial_{\beta} \Delta^{2}\left(\beta, \omega_{D}, g\right)=\frac{\int_{0}^{\hbar \omega_{D}} \frac{d \epsilon}{\cosh ^{2} \frac{\beta E}{2}}}{\int_{0}^{\hbar \omega_{D}} \frac{d \epsilon}{E^{3}}\left(\tanh \frac{\beta E}{2}-\frac{\beta E}{2 \cosh ^{2} \frac{\beta E}{2}}\right)}
$$

Taking the limit $T \rightarrow T_{c}, \boldsymbol{\Delta} \rightarrow 0$, we obtain

$$
0<a^{2}=\frac{2\left(k_{B} T_{c}\right)^{2} \tanh \frac{\hbar \omega_{D} \beta_{c}}{2}}{\int_{0}^{\hbar \omega_{D} \beta_{c}} \frac{d x}{x^{3}}\left(\tanh \frac{x}{2}-\frac{x}{2 \cosh ^{2} \frac{x}{2}}\right)}<\infty
$$

implying $\alpha=\mathbf{1} / \mathbf{2}$. Notice that the above integrand is finite at $x=0$.

As illustration we evaluate the integral for $\hbar \omega_{D} \beta_{c}=10$ to get

$$
\Delta(T)=3.10 \cdot k_{B} T_{c}\left(1-\frac{T}{T_{c}}\right)^{\frac{1}{2}}, T \lesssim T_{c} .
$$

\section{References}

[1] A.L. Fetter and J.D. Walecka, Quantum Theory of Many-Particle Systems (McGraw-Hill Company, New York, 1971). 
[2] A. Atland and B. Simon, Condensed Matter Field Theory (Cambridge University Press, Cambridge 2010).

[3] E.C. Marino, Quantum Field Theory Approach to Condensed Matter Physics (Cambridge University Press, Cambridge, 2017).

[4] J. Bardeen, L.N. Cooper and J.R. Schrieffer, Phys. Rev. 106, 162 (1957). 